\title{
Patient Preferences for Topical Psoriasis Treatments are Diverse and Difficult to Predict
}

Lars Iversen · Henny B. Jakobsen

Received: March 1, 2016 / Published online: April 28, 2016

(C) The Author(s) 2016. This article is published with open access at Springerlink.com

\section{ABSTRACT}

Introduction: Topicals are a mainstay in psoriasis vulgaris treatment and are used concomitantly even in patients receiving systemic therapy. Patient acceptance of topical treatment can impact adherence and, consequently, real-life effectiveness. This study aimed to identify patient preferences for topical treatment attributes and to evaluate patient acceptability of topical treatments.

Methods: This 5-day study evaluated three inactive presentations: fixed combination calcipotriol/betamethasone dipropionate (Cal/ BD) ointment vehicle in a tube, Cal/BD gel vehicle in a bottle and $\mathrm{Cal} / \mathrm{BD}$ gel vehicle in a new Applicator delivery system. Participants with psoriasis on $\geq 2$ locations were recruited, aiming for equal proportions in the categories: male/female; $<40 / \geq 40$ years old; working/not

Enhanced content To view enhanced content for this article go to http://www.medengine.com/Redeem/ A2C4F060169BA8D3.

L. Iversen

Aarhus University Hospital, Aarhus, Denmark

H. B. Jakobsen $(\square)$

LEO Pharma A/S, Ballerup, Denmark

e-mail: henny.jakobsen@leo-pharma.com working. Participants ranked a predefined 'wish list' of 13 topical treatment attributes, applied each product and evaluated them in a user survey, indicating how well the products delivered on 16 statements matching the 13 attributes. Afterward, participants indicated preference by psoriasis plaque location.

Results: Patient preferences differed to the extent that the proportion rating an attribute of high importance was similar to the proportion rating the same attribute of low importance. All attributes received similar mean ranking scores. Analysis by demographic characteristics revealed preferences are not associated with gender, age or employment status. Participants did not perceive much difference between the gel and ointment, but expressed preferences for different presentations to treat different plaque locations. The gel Applicator was preferred for 8/14 locations and both gel presentations were strongly preferred for hairy scalp.

Conclusions: Patients with psoriasis have diverse preferences, which cannot be predicted by demographics. Individual patient choice should be considered to increase the acceptability and a range of topical 
formulations should be available to support treatment adherence and improve clinical outcomes.

Funding: LEO Pharma A/S, Ballerup, Denmark.

Keywords: Applicator device; Individualizing therapy; Patient preference; Psoriasis; Survey; Topical treatments; Vehicle

\section{INTRODUCTION}

Psoriasis vulgaris is a chronic, inflammatory skin disorder [1]. It is associated with an increased risk of serious physical comorbidities and psychosocial symptoms, which contribute to a considerable burden of disease that can significantly reduce patient quality of life [2]. In addition, a substantial burden can be attributed to psoriasis treatment $[3,4]$. Nearly $80 \%$ of psoriasis cases are of mild-to-moderate severity and can be managed by topical therapy alone [5]. However, adherence is often poor [6] and up to $50 \%$ of prescriptions for topical therapies are never filled [7]. The most frequent challenges associated with topical treatment adherence include patient acceptability of vehicle (mainly related to treatment being messy and/ or time consuming) and patient perception of effectiveness $[4,8,9]$. In all therapy areas, poor adherence is a key limiting factor for real-world treatment effectiveness [10]. Various strategies have been employed to increase adherence, for example, slow-release formulations of analgesics for treatment of chronic pain to reduce dosing frequency, taste adjustment of liquids for oral intake by small children, depot injections of contraceptives and rapid-acting insulin. In patients with psoriasis, the importance of identifying and addressing individual wishes and preferences regarding formulation, administration and application of topical treatments has been widely recognized [11].

Fixed combination calcipotriol $50 \mu \mathrm{g} / \mathrm{g}$ (Cal) and betamethasone $0.5 \mathrm{mg} / \mathrm{g}$ (as dipropionate; BD) gel and ointment formulations are effective and well-tolerated topical therapies for psoriasis vulgaris [5]. As the two active components are incompatible in aqueous and alcoholic media, both ointment and gel vehicles are lipophilic and contain no alcohol or water [12]. The vehicle compositions provide good emollient properties that help to induce better hydration of dry skin and skin barrier repair [13, 14], but this inevitably means that both vehicles can be perceived as greasy. Recently, the Cal/BD gel Applicator was developed in collaboration with psoriasis patients and health-care professionals to provide a topical treatment delivery system that caters to unmet patient needs, including targeted application, the ability to replicate the dispensed amount of gel from day to day and a 'no touch' option enabling patients and carers to apply treatment without getting gel on their fingers [15].

Here, we present a study focusing on patient acceptability of topical therapies without interference from effectiveness, comparing three inactive presentations of topical treatment vehicles. The study aimed to identify associations between patient preferences for different attributes and demographic characteristics.

\section{METHODS}

\section{Participants}

Participants aged $\geq 18$ years with a clinical diagnosis of psoriasis vulgaris affecting the scalp plus at least one other skin area, who used prescription psoriasis topical treatments 
during the previous year, were recruited. We wanted to maximize the number of participants with scalp psoriasis. The scalp is a commonly affected and inconvenient application site that patients dislike treating, so it is important to gain patient insight into treating this area. Participants were excluded if they were allergic to any fragrance-free topical products. To enable comparison across demographic categories, participants were selectively recruited with the aim of having equal proportions in the following categories: male/ female; $<40 / \geq 40$ years of age; working/not working.

\section{Development of a Topical Treatment Attribute List and User Survey}

Literature on patient preferences for topical psoriasis treatment formulations and previous market research (LEO Pharma) on burden of psoriasis treatment was used to identify 13 topical treatment attributes that, in addition to treatment efficacy, safety and cost, are important to patients [3, 14, 16-19]. Based on these attributes plus the target attributes for the new gel Applicator [15], a user survey comprising 16 statements was developed against which participants evaluated the three treatment presentations. Some statements were deliberately presented in their negative form to enhance careful reading and consideration of responses. One topical treatment attribute, spreadability, was not reflected in the survey due to the fact that the dedicated spreading surface of the Applicator could confuse responders as to what they were asked to evaluate-the usefulness of the spreading surface or the inherent spreadability of the gel (Table 1).

\section{Study Design and Objectives}

Participants attended two sessions conducted 5 days apart at a non-clinic location (Fig. 1). During the first instruction session, participants ranked topical treatment attributes from the provided 13-attribute list during a card-sorting exercise (scale $1-13$, where 1 is of the highest importance and 13 is of the least importance). Participants were provided with three inactive topical treatments and instructions for use: $\mathrm{Cal} /$ BD ointment vehicle in an aluminum tube; $\mathrm{Cal} /$ $\mathrm{BD}$ gel vehicle in a plastic bottle; and Cal/BD gel vehicle in the Applicator (pre-assembled and primed). They were also provided with a user survey for each treatment presentation and survey completion instructions. On each day of testing, participants applied one treatment presentation to at least one area affected by psoriasis; all participants tested all presentations in an order of their own choice. Participants graded the treatment presentation against the 16 user survey statements on a seven-point scale: totally disagree (1), largely disagree (2), slightly disagree (3), neither disagree nor agree (4), slightly agree (5), largely agree (6) and totally agree (7). During the second feedback session, participants indicated their preference for each of the three topical treatment presentations ( 1 is first choice and 3 is last choice, or not relevant) in relation to their plaques in the following locations of relevance to them: scalp (hairy/bald), face, neck, chest/ stomach (hairy/non-hairy), back/buttocks (hairy/non-hairy), arms (hairy/non-hairy), legs (hairy/non-hairy), hands and ankles/feet.

The study objectives were to determine patient preferences for different topical treatment attributes and to identify patient characteristics that could help guide topical 
Table 1 Topical treatment attributes and statements in the user survey

\section{Topical treatment attribute}

It is easy to get the product out of the container

I can control the amount I get out of the container

It is easy to apply only where I want it

It is not greasy on the skin

It does not make the hair greasy

It does not smell/is odorless

It is not seen on the skin after application

I do not need to touch the product with my fingers

It does not create a mess around me

The time it takes to apply is acceptable

It is quick to absorb into the skin

I can use the same product for all affected skin areas

It is easy to spread onto the skin

\section{User survey statement}

I can easily get the product out of the container

I do not feel to be in control of the applied amount when I use the product ${ }^{\mathrm{a}}$ It is easy to apply the product only where I want it

I can avoid wasting any product

The content is not greasy on my skin

The content makes my hair greasy ${ }^{\mathrm{a}}$

The product does not smell

The product can be seen on my skin ${ }^{\mathrm{a}}$

I need to touch the product with my fingers ${ }^{\mathrm{a}}$

Things do not get messy around me when I use the product

The time it takes to apply the product is acceptable

The time for the product to be absorbed into the skin so that I can get dressed is acceptable

I cannot use this product for all my affected skin areas ${ }^{\mathrm{a}}$

With this product, it does not matter if there is hair in the area of application I cannot reach to apply the product to all skin areas affected by psoriasis ${ }^{\mathrm{a}}$

I experience a pleasant sensation in the affected skin area right after I apply the product

\footnotetext{
a Statements were inverted prior to data analysis
}

treatment prescription, thereby maximizing patient acceptance.

Data collection complied with the 2014 version of the Legal and Ethical Guidelines for Healthcare Market Research developed by the Ethics Team of the British Healthcare Business Intelligence Association, the UK Data Protection Act 1998 and Directive 95/46/EC on the protection of individuals with regard to the processing of personal data and on the free movement of such data. Independent Ethics Committee approval is not required for market research in the UK. Informed consent was obtained from all patients for being included in the study.

\section{Statistical Analysis}

Standard descriptive statistical methods were used as the primary method of data analysis (SAS 9.4, SAS Institute, Inc., Cary, NC, USA). Multiple logistic regression analysis was used to screen for associations between participant characteristics and topical treatment 


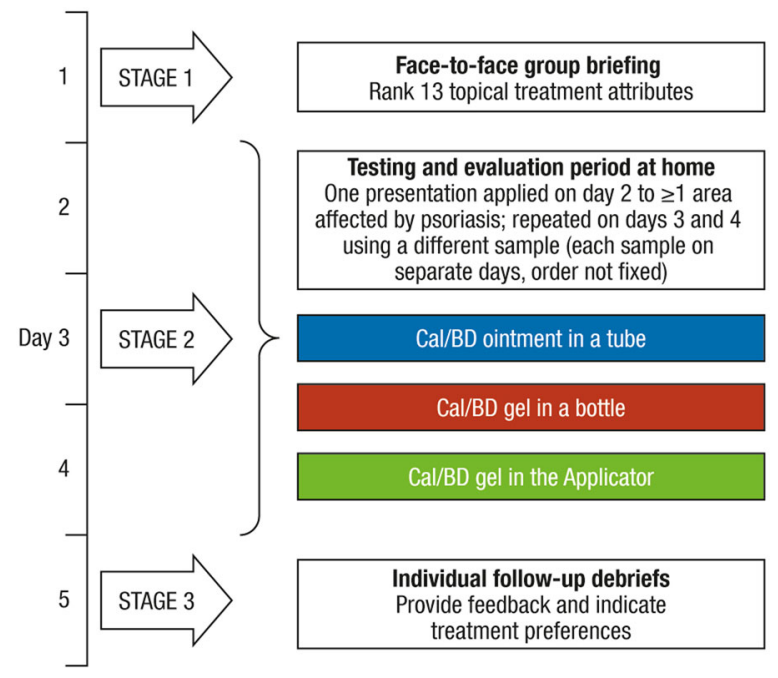

Fig. 1 Study design. Cal/BD, calcipotriol $50 \mu \mathrm{g} / \mathrm{g}$ and betamethasone $0.5 \mathrm{mg} / \mathrm{g}$ (as dipropionate)

preference by plaque location. Initially, it was planned that participants would be classified as working/not working, but prior to analysis, participants were re-classified as working full time or not working/not working full time. This was considered more relevant from a 'burden of treatment' perspective, since part-time work would allow more flexibility and time for topical treatment application.

\section{RESULTS}

\section{Participants}

One hundred and twelve participants were recruited and all completed the study (Table 2). Briefly, the study was performed in two parts: The first group participated from 27 November to 2
December 2014 and the second group 22 to 27 January 2015. Recruitment started in October 2014; participants were recruited by Medicys Ltd through health-care professionals (68\%), the Medicys database (21\%) and external recruiters with their own contacts (11\%). The median age was between 40 and 49 years for both males and females and $49 \%$ of the participants had psoriasis for 15 years or more (Table 3). Most participants (94\%) experienced face-to-face contact all or a lot of the time in their daily life, and $67 \%$ had such contact at leisure time.

Participants had psoriasis on up to 11 skin areas and $80 \%$ reported having at least one affected area where they found application of topical treatment difficult (Fig. 2a, b). Upon recruitment, participants were required to have both scalp and at least one other skin area affected by psoriasis. However, at the time of the study, $12 \%$ of participants reported not having scalp psoriasis; these patients were not excluded from the analysis, so as not to waste their contributions with regard to their general priorities and evaluation of the products on other skin areas.

\section{Participant Prioritization of Topical Treatment Attributes}

In the initial card-sorting exercise, all 13 topical treatment attributes received similar mean ranking scores (range 6.0-8.1; scale 1-13), indicating that participants had very different priorities. In most cases, the proportion of

Table 2 Participant distribution across strata

\begin{tabular}{|c|c|c|c|c|c|}
\hline & \multicolumn{2}{|l|}{ Men } & \multicolumn{2}{|l|}{ Women } & \multirow[t]{2}{*}{ Total } \\
\hline & $<40$ years & $\geq 40$ years & $<40$ years & $\geq 40$ years & \\
\hline Working full time, $n$ & 16 & 11 & 13 & 9 & 49 \\
\hline Not working or not working full time, $n$ & 8 & 19 & 14 & 22 & 63 \\
\hline Total, $n$ & 24 & 30 & 27 & 31 & 112 \\
\hline
\end{tabular}


Table 3 Participant age distribution and duration of psoriasis

Proportion of participants (\%)

Age range, years

$\begin{array}{rr}18-29 & 19.6 \\ 30-39 & 25.9 \\ 40-49 & 25.9 \\ 50-59 & 14.3 \\ 60-69 & 12.5 \\ 70-79 & 1.8\end{array}$

Duration of psoriasis, years

$$
\begin{array}{ll}
<5 & 17.0 \\
5 \text { to }<15 & 33.9 \\
\geq 15 & 49.1
\end{array}
$$

participants who indicated an attribute was of high importance (i.e., ranked $1-3$ of 13) was similar to the proportion who indicated the same attribute was of low importance (i.e., ranked $11-13$ of 13 ; Fig. 3 ). However, there was a numerical difference between participant rankings for some attributes. For example, the fact that participants did not need to touch the product (mean ranking score 6.0) was considered of high importance by $29 \%$ of participants, while $14 \%$ considered this attribute to be of low importance (Fig. 3). Attributes that relatively few patients considered of neither high nor low importance, such as 'I can use the same product for all affected areas', were concluded to be of medium priority overall.

\section{Participant Prioritization of Topical Treatment Attributes by Participant Characteristics}

When analyzed by gender, age or employment status, little difference was observed between
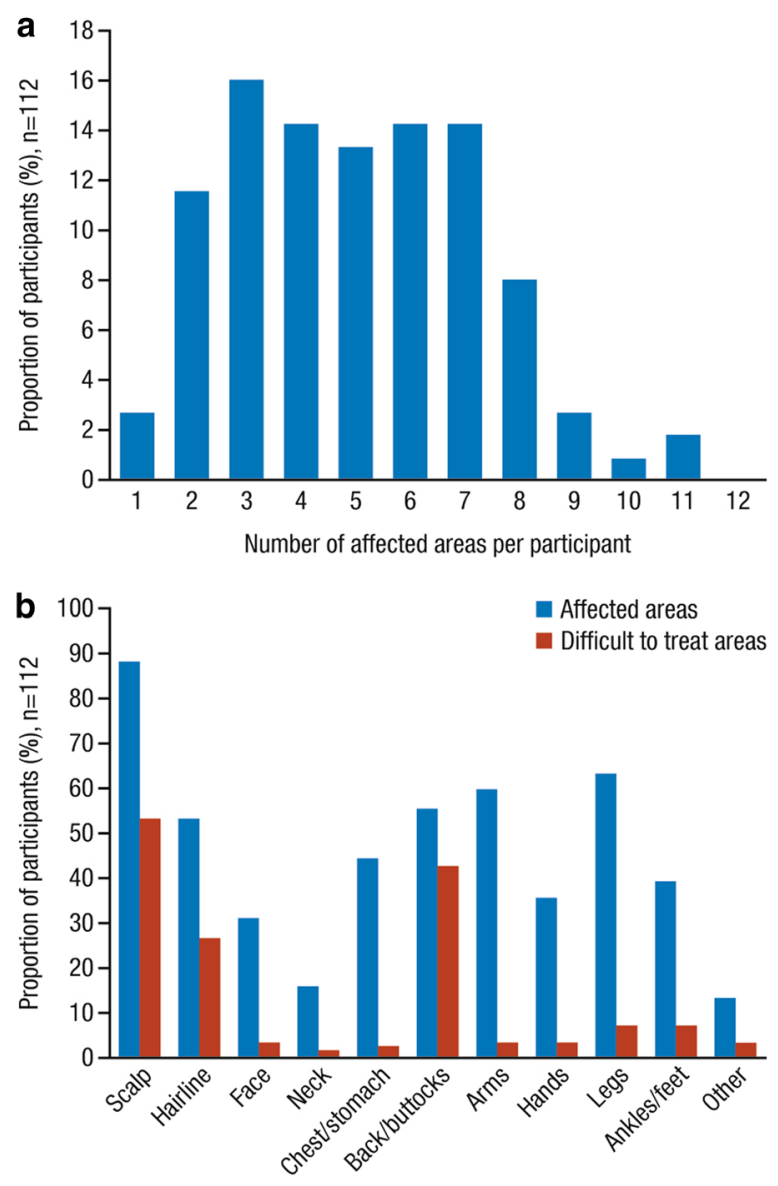

Fig. 2 a Number of psoriasis affected skin areas per participant; $\mathbf{b}$ location of psoriasis and participant assessment of whether these locations are difficult to treat

the proportion of participants rating the same attribute as of low and high importance. However, numerical differences were observed for some attributes. Of note, the product being easy to spread onto the skin was of higher importance (i.e., ranked 1-3) to females (31\%) than males (11\%), and the fact that the product was not greasy on the skin was of higher importance to participants who worked full time (39\%) than those who did not (18\%). Precise application ('only where I want it') was considered of higher importance to participants under 40 years of age (33\%) than older participants (18\%), and being able to get the product out of the container easily was 


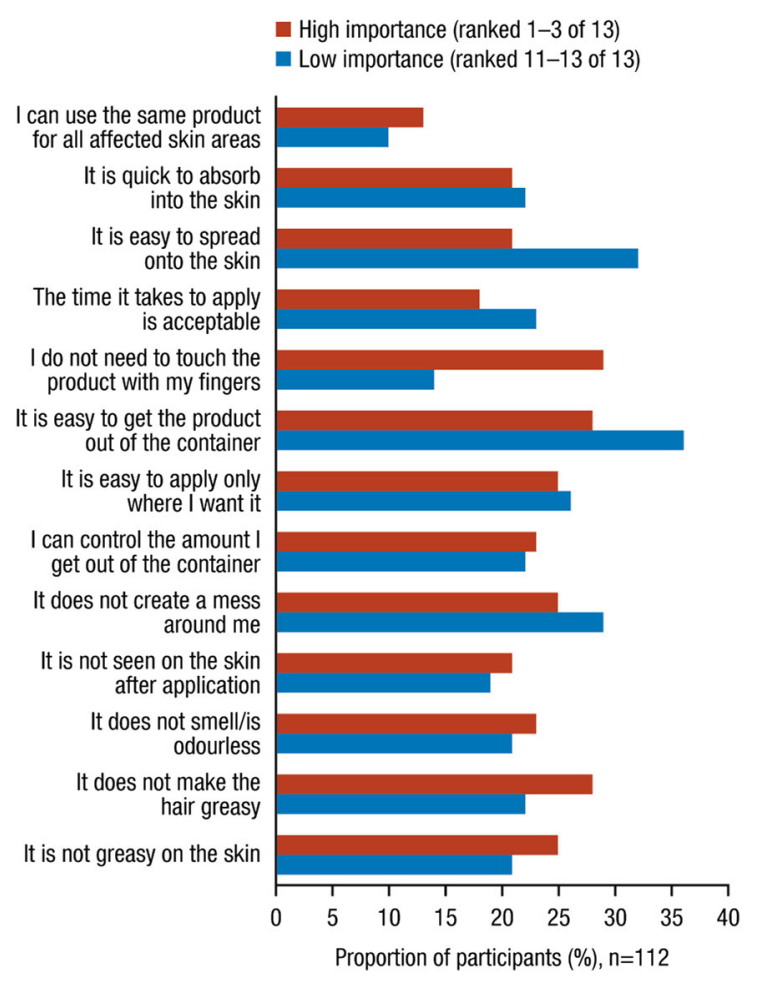

Fig. 3 Topical treatment attributes of high and low importance to participants

considered of higher importance to those who worked full time (28\%) than those who did not (13\%). More males (28\% vs $14 \%$ of females) and more participants aged over 40 years ( $26 \%$ vs $14 \%$ of participants aged less than 40 years) indicated it was important that the product was absorbed quickly into the skin. Additionally, an acceptable time taken to apply the product was of higher importance to participants who worked full time (22\% vs $11 \%$ of participants not working or working part time).

\section{Participant Evaluation of Topical Treatment Presentations}

Participants' mean score ratings of the three presentations indicated that the gel presentations matched the topical treatment wish list attributes to a higher degree than the ointment and, in particular, more participants scored the gel Applicator the highest (Fig. 4a, b). For most topical treatment attributes, the participant ratings increased stepwise from the ointment in a tube, to gel in a bottle, to gel in the Applicator (Fig. 4a). A larger proportion of participants largely or totally agreed (score of 6 or 7 on a scale of 1-7) that they can avoid wasting the product when using the gel Applicator compared with the gel in a bottle and the ointment in a tube (Fig. 4a, b). More participants largely or totally agreed it is easy to get the ointment out of the tube compared with getting the gel out of the bottle or the gel Applicator (Fig. 4a, b). Additionally, more participants largely or totally agreed the gel Applicator allows them to apply the product without having to touch it with their fingers compared with the gel in a bottle and the ointment (Fig. 4a, b). Out of the $29 \%$ of participants for whom the 'no touch' attribute was of high importance, 30\% largely or totally agreed that the Applicator provided this option.

Additional analysis was performed to evaluate the extent to which each treatment presentation delivered on each attribute (on a scale of $1-7$, where 1 is totally disagree and 7 is totally agree) using data only from those participants who classified the attribute as most important to them (ranking score 1-3). There were no clear differences between this subgroup and the overall study population.

\section{The Impact of Pain or Reduced Strength or Mobility in Hands}

Of the 112 participants, 27 (24\%) reported pain, reduced strength or reduced mobility in their hands. For the attributes: 'I can easily get the 
With this product it does not matter if there is hair in the area

I can use this product for all my affected skin areas

The time for the product to be absorbed into the skin so that I can get dressed is acceptable

The time it takes to apply the product is acceptable

I can reach to apply the product to all skin areas affected by psoriasis

I do not need to touch the product with my fingers

I can easily get the product out of the container

It is easy to apply the product only where I want it

I feel in control of the applied amount when I use the product

Things do not get messy around me when I use the product

The product cannot be seen on my skin

The product does not smell

The content does not make my hair greasy

The content is not greasy on my skin

I experience a pleasant sensation in the affected skin area right after I apply the product
Fig. 4 a Participant evaluation of the degree to which each treatment presentation delivered on the topical treatment attribute (mean agreement score, range 1-7) and $\mathbf{b}$ the

product out of the container'; 'I feel in control of the applied amount'; 'I can reach to apply the product to all skin areas affected'; 'It is easy to apply the product only where I want it'; and 'I can use this product for all my affected skin areas', analysis of mean rating scores showed no differences between participants with pain or reduced strength or mobility in their hands and the rest of the population.
Gel in Applicator $\quad$ Gel in bottle $\quad$ Ointment in tube
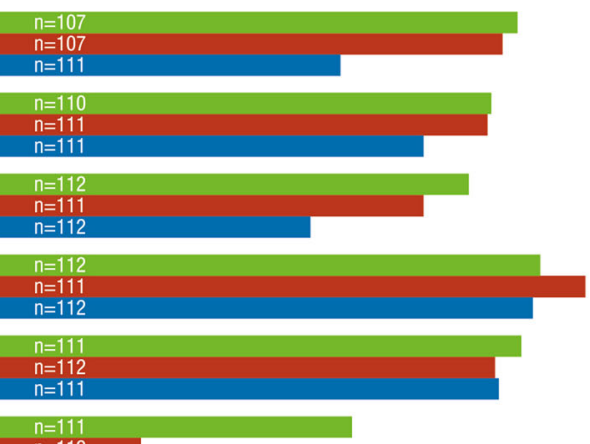

$n=112$
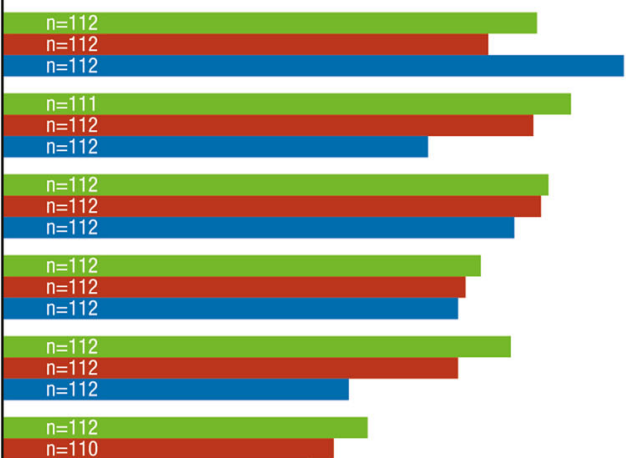

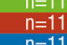

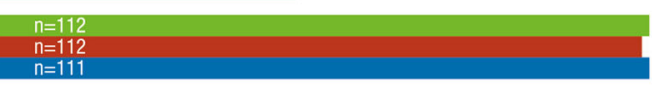

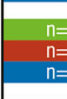

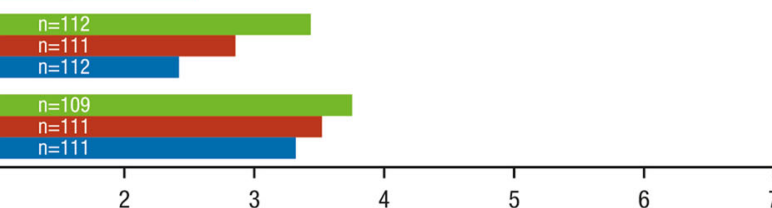

Mean agreement score proportion of patients who largely or totally agreed to the treatment presentation delivered on the topical treatment attributes

\section{Preferences for Topical Treatment by Affected Skin Area}

Participants indicated which topical treatment they preferred for each plaque location of relevance to them. Overall, the gel Applicator was preferred for 8/14 plaque locations, while the ointment was preferred for 5/14 locations

(Fig. 5). The two gel presentations combined 


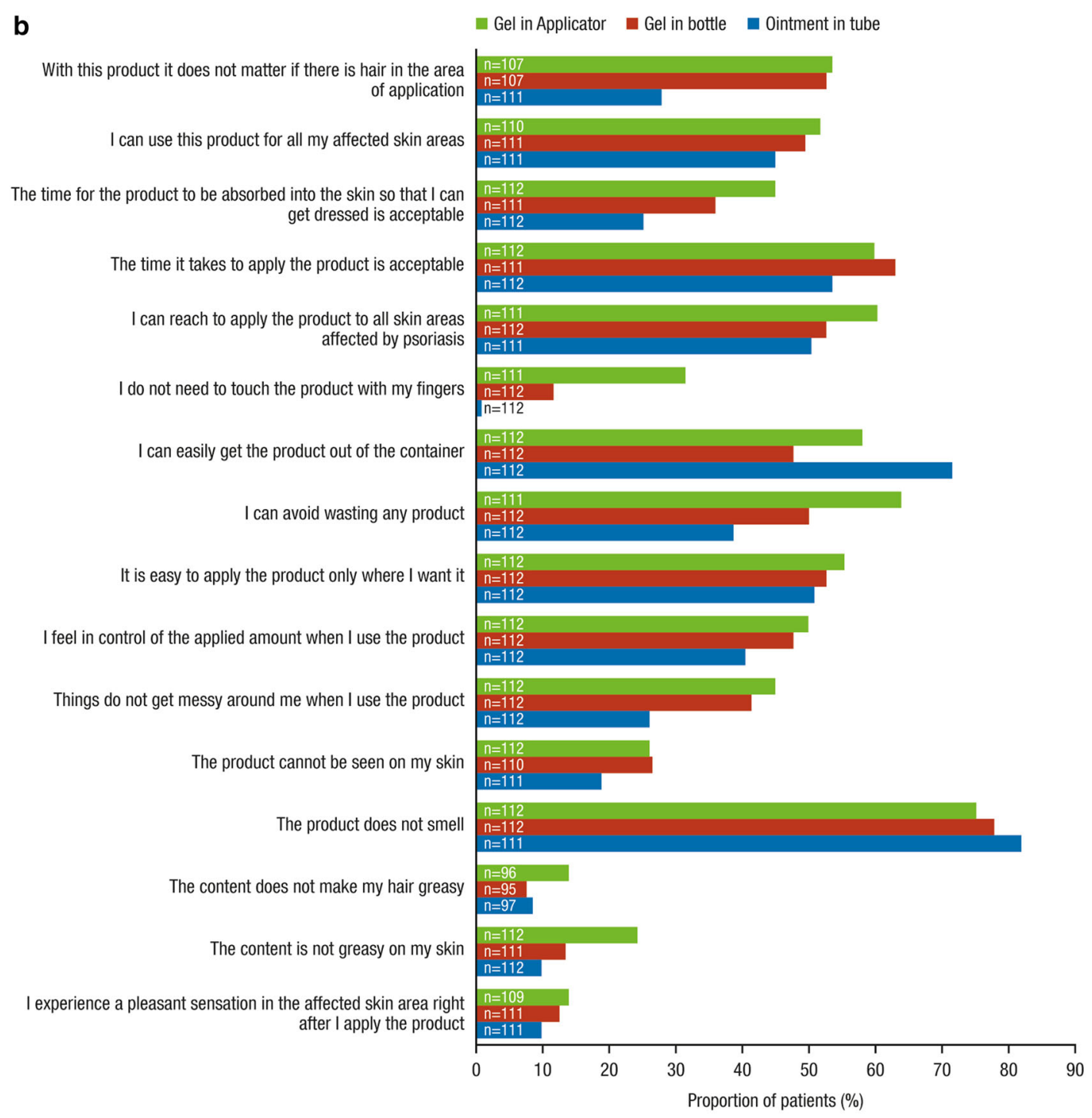

Fig. 4 continued

were preferred for all locations except the neck (ointment preferred by 52\%) and hairy back/ buttocks (50\%). Using the gel Applicator as reference and adjusting for the other participant characteristics, none of the characteristics analyzed (gender, age, work status and hand pain/impairment) showed a strong independent association with patient preference. For treatment of non-hairy back/ buttocks, female gender and working full time were independently associated with lower preference for ointment in tube versus gel in Applicator [females: odds ratio (OR) $0.14 ; 95 \%$ confidence interval (CI) 0.02-0.95; working full time: OR 0.06; 95\% CI 0.01-0.59]. For treatment of hands, female gender and working full time were independently associated with lower preference for both ointment in tube (females: OR 0.07; 95\% CI 0.008-0.50; working full time: OR $0.1 ; 95 \%$ CI $0.01-0.95)$ and gel in bottle (females: OR 0.02; 95\% CI 0.001-0.23; working full time: OR 0.043; 95\% CI $0.003-0.66$ ) 


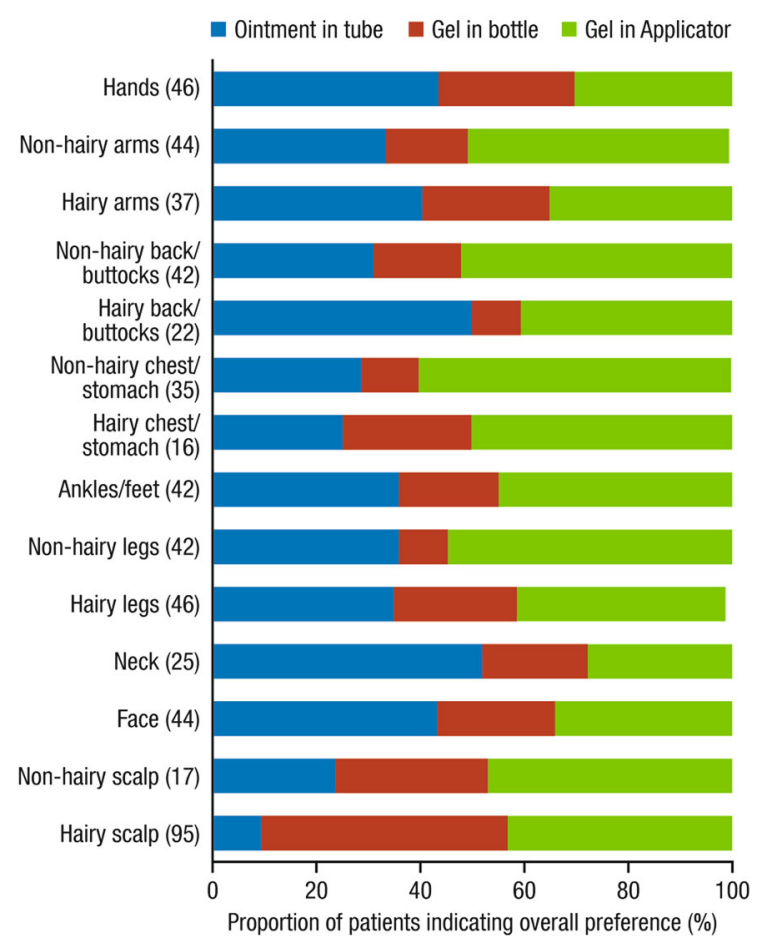

Fig. 5 Overall participant preferences for topical treatment of affected skin area. The number of respondents is indicated in brackets

compared with gel in Applicator. Of note, no presentation was consistently preferred for treatment of hairy versus non-hairy areas. For treatment of psoriasis on a hairy scalp, there was almost equal preference for each of the two gel presentations (bottle 47\%; gel Applicator 44\%), and very low preference for ointment (9\%). Among participants who had applied the gel presentations to hairy scalp, there were no participant characteristics (gender, age, working status, hand pain or hand impairment) independently associated with preference for either treatment presentation.

\section{DISCUSSION}

This patient preference study indicates that patients with psoriasis vulgaris are diverse in their priorities for topical treatment and express a preference for different presentations to treat different plaque locations. There is no 'one fits all' approach to the treatment of psoriasis, highlighting the importance of 'personalized medicine' and consideration of patient needs when deciding treatment regimens.

The attributes evaluated in this study were selected based on well-established formulation-based barriers for topical medication use, combined with patient input obtained prior to and during development of the Applicator. Overall, participants indicated the gel and ointment presentations met the desired topical treatment attributes to a similar degree. All tested presentations received relatively low scores for some of the attributes, especially attributes related to greasiness and absorption, which was expected given the lipophilic vehicles of the Cal/BD formulations. This is a particular challenge for $\mathrm{Cal} / \mathrm{BD}$ topical products [12].

Adherence to topical treatments is an ongoing concern in psoriasis therapy and it has been recognized that the patient's perspective should be considered to improve both physiological and psychological aspects of psoriasis management [11]. Indeed, many efforts have been made to educate and motivate patients to improve treatment adherence, such as development of a topical treatment optimization program to improve the information given to the patients and to result in an engaged patient-physician relationship [20], and mobile phone-based interventions, which have been investigated in a pilot study in patients receiving systemic psoriasis treatment [21]. With the patient perspective in mind, the gel Applicator was developed with the intention to design a topical treatment option targeting selected patient wishes, namely control over the amount of medication applied, precise application and being able to avoid touching the product with fingers, with the aim of 
improving treatment adherence [15]. This study suggests that the gel Applicator does meet the wishes of some patients. One-third of participants indicated a 'no touch' treatment option was one of the most important topical treatment attributes; one-third of these and one-third of all participants largely or totally agreed that the gel Applicator provided them with this option. This confirms observations made during one of the patient development sessions for the gel Applicator [15]. However, despite the gel Applicator spreading surfaces, many participants commented that they still applied the product with their fingers. This is to be expected, as patients have been applying topical therapies and ensuring the right amount of treatment is administered using their fingers for many years.

The diverse patient priorities observed in this study, and the indication that patients would prefer to use different treatment presentations for different plaque locations, suggests that offering a number of different topical treatment solutions may improve patient acceptance. For example, Cal/BD gel was developed to offer a less greasy treatment option than Cal/BD ointment, particularly for patients with psoriasis on the scalp [22], which this study confirms is a difficult-to-treat area. This study indicates that the gel presentations were strongly preferred over ointment for treatment of this area. However, it is also clear that the treatment of hairy scalp remains challenging.

The study was conducted as market research, meaning that there could be no interference with treatment of the skin disease; thus, the study had to be very brief and the duration was limited to 5 days, with the requirement that participants tried each sample at least once. This exposure does not compare with real-life treatment of a chronic skin disease and does not allow for analysis of patient preferences for vehicle and/or delivery system over the long term. However, given the chronic nature of psoriasis and the reported duration since diagnosis, most participants were expected to have broad experience with topical treatments and to be able to provide their opinion on a presentation relatively quickly; however, there may have been too little time for participants to evaluate a new type of presentation like the gel Applicator. The short-term experience with the Applicator may underestimate its usability, which may improve with practice. Observational clinical studies with active treatments in real-life treatment conditions are required to draw conclusions on whether patient preference translates into greater treatment adherence and increased real-life effectiveness.

\section{CONCLUSION}

Patients with psoriasis have very different priorities, which cannot be predicted from simple demographics. Individuals should be informed about their options and consulted on their personal preferences during decisions on their topical treatment regimen; preferably, more than one formulation should be made available to patients for use on different plaque locations. In terms of patient preferences identified by this study, the gel formulation is preferred over ointment by most patients for psoriasis on a hairy scalp, and the gel Applicator is preferred for more plaque locations. Giving patients with psoriasis a choice in the formulation and delivery system of their topical treatment, thereby meeting some of their individual preferences, may improve adherence by increasing the acceptability of the treatment they use in their daily life. 


\section{ACKNOWLEDGMENTS}

Sponsorship and article processing charges for this study were funded by LEO Pharma A/S, Ballerup, Denmark. We would like to thank Bridget Pumfrey and Matt Brooks from Brooks Pumfrey Research Limited, London, UK, for carrying out the study. Statistical support was provided by René Depont Christensen from Bio Stata ApS, Birkerød, Denmark, funded by LEO Pharma. All named authors meet the International Committee of Medical Journal Editors (ICMJE) criteria for authorship for this manuscript, take responsibility for the integrity of the work as a whole, and have given final approval for the version to be published. Additionally, we thank Catherine Risebro, PhD, from Mudskipper Business Limited, London, UK, who provided medical writing support funded by LEO Pharma.

Disclosures. H Jakobsen is an employee of LEO Pharma A/S. L Iversen has served as a consultant and/or paid speaker for and/or participated in clinical trials sponsored by companies that manufacture drugs used for the treatment of psoriasis, including AbbVie, Almirall, Amgen, Celgene, Centocor, Eli Lilly, Janssen-Cilag, LEO Pharma, Merck Sharp and Dohme, Novartis and Pfizer.

Compliance with Ethics Guidelines. Data collection complied with the 2014 version of the Legal and Ethical Guidelines for Healthcare Market Research developed by the Ethics Team of the British Healthcare Business Intelligence Association, the UK Data Protection Act 1998 and Directive 95/46/EC on the protection of individuals with regard to the processing of personal data and on the free movement of such data. Independent Ethics Committee approval is not required for market research in the UK.
Informed consent was obtained from all patients for being included in the study.

Open Access. This article is distributed under the terms of the Creative Commons Attribution-NonCommercial 4.0 International License (http://creativecommons.org/licenses/ by-nc/4.0/), which permits any noncommercial use, distribution, and reproduction in any medium, provided you give appropriate credit to the original author(s) and the source, provide a link to the Creative Commons license, and indicate if changes were made.

\section{REFERENCES}

1. Schön MP, Boehncke W-H. Psoriasis. N Engl J Med. 2005;352:1899-912.

2. Krueger G, Koo J, Lebwohl M, et al. The impact of psoriasis on quality of life: results of a 1998 National Psoriasis Foundation patient-membership survey. Arch Dermatol. 2001;137:280-4.

3. Bewley A, Burrage DM, Ersser SJ, et al. Identifying individual psychosocial and adherence support needs in patients with psoriasis: a multinational two-stage qualitative and quantitative study. J Eur Acad Dermatol Venereol. 2014;28:763-70.

4. Fouéré S, Adjadj L, Pawin H. How patients experience psoriasis: results from a European survey. J Eur Acad Dermatol Venereol. 2005;19(Suppl 3):2-6.

5. Menter A, Korman NJ, Elmets CA, et al. Guidelines of care for the management of psoriasis and psoriatic arthritis. Section 3. Guidelines of care for the management and treatment of psoriasis with topical therapies. J Am Acad Dermatol. 2009;60:643-59.

6. Thorneloe RJ, Bundy C, Griffiths CE, et al. Adherence to medication in patients with psoriasis: a systematic literature review. $\mathrm{Br} J$ Dermatol. 2013;168:20-31.

7. Storm A, Andersen SE, Benfeldt E, et al. One in 3 prescriptions are never redeemed: primary nonadherence in an outpatient clinic. J Am Acad Dermatol. 2008;59:27-33.

8. van de Kerkhof PC, de Hoop D, de Korte J, et al. Patient compliance and disease management in the 
treatment of psoriasis in the Netherlands. Dermatology. 2000;200:292-8.

9. Devaux S, Castela A, Archier E, et al. Adherence to topical treatment in psoriasis: a systematic literature review. J Eur Acad Dermatol Venereol. 2012;26(Suppl 3):61-7.

10. Naderi SH, Bestwick JP, Wald DS. Adherence to drugs that prevent cardiovascular disease: meta-analysis on 376,162 patients. Am J Med. 2012;125:882-7.

11. Zeichner JA, Lebwohl MG, Menter A, et al. Optimizing topical therapies for treating psoriasis: a consensus conference. Cutis. 2010;86(3 Suppl):5-31.

12. Simonsen L, Hoy G, Didriksen E, et al. Development of a new formulation combining calcipotriol and betamethasone dipropionate in an ointment vehicle. Drug Dev Ind Pharm. 2004;30:1095-102.

13. Fluhr JW, Cavallotti C, Berardesca E. Emollients, moisturizers, and keratolytic agents in psoriasis. Clin Dermatol. 2008;26:380-6.

14. Bewley A, Page B. Maximizing patient adherence for optimal outcomes in psoriasis. J Eur Acad Dermatol Venereol. 2011;25(Suppl 4):9-14.

15. Rasmussen G, Bech LL, Nielsen TW. An applicator delivery system for fixed-combination calcipotriene plus betamethasone dipropionate topical suspension (gel): innovating psoriasis vulgaris treatment through patient collaboration. Dermatol Ther (Heidelb). 2015;5:235-46.
16. Eastman WJ, Malahias S, Delconte J, et al. Assessing attributes of topical vehicles for the treatment of acne, atopic dermatitis, and plaque psoriasis. Cutis. 2014;94:46-53.

17. Housman TS, Mellen BG, Rapp SR, et al. Patients with psoriasis prefer solution and foam vehicles: a quantitative assessment of vehicle preference. Cutis. 2002;70:327-32.

18. Augustin M, Radtke MA, Zschocke I, et al. The patient benefit index: a novel approach in patient-defined outcomes measurement for skin diseases. Arch Dermatol Res. 2009;301:561-71.

19. Blome C, Augustin M, Behechtnejad J, et al. Dimensions of patient needs in dermatology: subscales of the patient benefit index. Arch Dermatol Res. 2011;303:11-7.

20. Reich K, Mrowietz U, Karakasili E, et al. Development of an adherence-enhancing intervention in topical treatment termed the topical treatment optimization program (TTOP). Arch Dermatol Res. 2014;306:667-76.

21. Balato N, Megna M, Di Costanzo L, et al. Educational and motivational support service: a pilot study for mobile-phone-based interventions in patients with psoriasis. $\mathrm{Br} \mathrm{J}$ Dermatol. 2013;168:201-5.

22. Papp K, Berth-Jones J, Kragballe K, et al. Scalp psoriasis: a review of current topical treatment options. J Eur Acad Dermatol Venereol. 2007;21:1151-60. 